\title{
Cancer patients treated with the Banerji protocols utilising homoeopathic medicine: A Best Case Series Program of the National Cancer Institute USA
}

\author{
PRASANTA BANERJI ${ }^{1}$, DONALD R. CAMPBELL ${ }^{2}$ and PRATIP BANERJI ${ }^{1}$ \\ ${ }^{1}$ PB Homoeopathic Research Foundation, Kolkata, West Bengal, India; \\ ${ }^{2}$ Saint Luke's Hospital and University of Missouri, Kansas City, MO, USA
}

Received February 21, 2008; Accepted April 23, 2008

\begin{abstract}
Although many studies have been conducted on the role of alternative medicine in the treatment of cancer, only a few reports have been published regarding the total regression of malignant tumors. At the PBH Research Foundation (PBHRF), two of the authors have used homoeopathic therapy to treat many patients with various malignant tumors. The objective of the present study was to have their treatment procedures evaluated and validated by the United States (US) National Cancer Institute (NCI) Best Case Series (BCS) Program. Lung and oesophageal carcinoma patients were treated with homoeopathic remedies at the PBHRF according to Banerji's protocol until there was complete regression of the tumors. Case records including pathology and radiology reports for 14 patients were submitted for review by the US NCI BCS Program. Four of these cases had an independent confirmation of the diagnosis and radiographic response and were accepted as sufficient information for the NCI to initiate further investigation. These four cases are presented in detail in this report along with follow-up and outcome information. This study describes the process and outcome of a selected case series review through the NCI BCS Program. The results of the review were deemed to be sufficient to warrant NCIinitiated prospective research follow-up in the form of an observational study.
\end{abstract}

\section{Introduction}

Homoeopathy is a medical system whose underlying principle is based on the theory that 'like cures like'. Homoeopathic medications are produced by using solutions of compounds which, when ingested by healthy volunteers, produce symptom complexes that mimic various diseases e.g., ingesting quinine

Correspondence to: Dr Prasanta Banerji or Dr Pratip Banerji, PBH Research Foundation, 10/3/1 Elgin Road, Kolkata 700020, West Bengal, India

E-mail: pbhrfindia@dataone.in

Key words: homoeopathic medicines, lung and oesophageal cancers, National Cancer Institute Best Case Series from cinchona bark induces symptoms similar to malaria (1). These solutions are serially diluted and succussed until the desired potency is produced. According to the World Health Organization (WHO), homoeopathy is the second most used medical system internationally, accounting for more than $\$ 1$ billion in costs. Twenty to thirty percent of French and German physicians use homoeopathy in clinical practice as cited by WHO, 2001. In Great Britain, 5 homoeopathic hospitals are part of the National Health System and $>30 \%$ of general practitioners use this type of treatment (2). In the United States, $>500$ physicians use homoeopathy in clinical practice and 5.5 million Americans use homoeopathic medicines (2-5).

Since 1991, the NCI has had a process for evaluating data from complementary and alternative medicine (CAM) practitioners that involves the same rigorous methods used in evaluating treatment responses with conventional medicine. This process, called the National Cancer Institute (NCI) Best Case Series (BCS) Program, provides an independent review of medical records, medical imaging and pathology materials from patients treated with unconventional cancer therapies (5). The Office of Cancer Complementary and Alternative Medicine (OCCAM) was established in October 1998 in order to coordinate and enhance the activities of the NCI in the arena of CAM. The Practice Assessment Program within OCCAM currently manages the NCI BCS Program $(6,7)$. Through this program, staff from OCCAM work with CAM practitioners to identify appropriate, well-documented cases. The primary goal of this program is to obtain and review sufficient information to determine if NCI-initiated research on a specific intervention is warranted.

\section{Patients and methods}

Homoeopathy, like every scientific and efficient system of medicine, has evolved through the years and the method used to treat these cases is by specific medicines in specific potencies as per what is known today as 'The Banerji Protocols'. Initially, we selected 14 cases among 300 cancer patients who had been treated at the PBH Research Foundation (PBHRF) clinic in Kolkata (Calcutta), India for these studies. Among these 14 cases, only 10 cases were selected for review by the BCS Program. At that time we had in our records 
Table I. Four cases of the PB Homoeopathic Research Foundation approved by the NCI Best Case Series Program.

\begin{tabular}{|c|c|c|c|c|c|}
\hline Case No. & Gender/age (y) & First clinic visit & Disease type & Treatment & $\begin{array}{l}\text { NCI BCS Program } \\
\text { review assessment }\end{array}$ \\
\hline 1 & Male/47 & $11 / 30 / 94$ & $\begin{array}{l}\text { Malignant non-small } \\
\text { cell lung carcinoma }\end{array}$ & $\begin{array}{l}\text { Kali Carbonicum } \\
200 \mathrm{c} 2 \text { drops thrice } \\
\text { in a week and Ferrum } \\
\text { Phosphoricum } \\
3 \times 2 \text { tablets } \\
\text { twice daily }\end{array}$ & Evaluable \\
\hline 2 & Female/77 & $12 / 27 / 94$ & $\begin{array}{l}\text { Small cell lung } \\
\text { carcinoma }\end{array}$ & $\begin{array}{l}\text { Kali Carbonicum } \\
\text { 200c a dose of } 2 \text { drops, } \\
\text { thrice in a week and } \\
\text { Ferrum Phosphoricum } \\
3 \times 2 \text { tablets twice daily }\end{array}$ & Evaluable \\
\hline 3 & Male/75 & $12 / 16 / 96$ & $\begin{array}{l}\text { Moderately } \\
\text { differentiated } \\
\text { squamous cell } \\
\text { oesophageal } \\
\text { carcinoma }\end{array}$ & $\begin{array}{l}\text { Condurango 30c } \\
\text { a dose of } 2 \text { drops, } \\
\text { twice daily }\end{array}$ & Evaluable \\
\hline 4 & Female/75 & 9/7/95 & $\begin{array}{l}\text { Squamous cell } \\
\text { oesophageal } \\
\text { carcinoma }\end{array}$ & $\begin{array}{l}\text { Condurango } 30 \mathrm{c} \\
\text { a dose of } 2 \text { drops, } \\
\text { twice daily }\end{array}$ & Evaluable \\
\hline
\end{tabular}

1260 cases of different types of cancers of which complete remission occurred in $\sim 21 \%$ of cases following our treatment. Examples of these responses can be reviewed by accessing an internet user group (http://health.groups.yahoo.com/group/ Ruta6/) maintained by brain tumor patients where follow-up of $>400$ brain tumor cases from different countries, who have been treated on Banerji protocols presently indicate that $>70 \%$ of patients are benefitting from treatment on our protocols. The slides were reviewed by a pathologist at the PBHRF. The selection criteria were as follows: a) recollection by PBHRF physicians that the patient had an improvement in his/her clinical condition concurrent with treatment at the PBHRF clinic; b) availability of the pathology report documenting the cancer diagnosis; and c) availability of medical imaging reports documenting an objective regression in tumor size. The relevant patient records were submitted to the OCCAM for review through the NCI BCS Program. An initial review by OCCAM of the documents from these cases indicated that these cases were eligible for further evaluation. The pathology slides and medical imaging studies, which were performed at the PBHRF, were requested and provided to the NCI for review. The histology and cytology slides were reviewed by a pathologist at the NCI Laboratory of Pathology and the medical imaging studies were reviewed by a radiologist in the Diagnostic Radiology Department (DRD) of the Warren G. Magnuson Clinical Center of the National Institutes of Health (NIH). After a complete review, these cases were presented at a meeting of the Cancer Advisory Panel for Complementary and Alternative Medicine (CAPCAM), a chartered committee advising the Director of the NIH and the Director of the Office of Alternative Medicine (OAM) on July 8, 1999.

\section{Results}

Summaries of the cases and relevant documentation from the 14 clinical patients who met the three selection criteria were submitted to the NCI and reviewed by the director of the OCCAM who is a medical oncologist. Table I summarizes the outcomes of the review of these cases. Further information and documentation was requested to clarify the clinical course of some patients. Four cases were found to have confirmed pathological diagnoses of cancer and adequate pre- and posttreatment medical imaging studies indicating a tumor response. These cases are described below.

Case No. 1. A 47-year-old man presented to the clinic on November 30, 1994. A previous chest X-ray (November 18, 1994) revealed a well-defined, large mass in the left upper mediastinum with well-expanded lung fields and an area of consolidation in the left upper lobe (Fig. 1A). A computed tomography (CT) scan (Fig. 1B) performed on Nov 19, 1994 showed an 8.0x6.4 cm well-defined, large, soft tissue mass of homogeneous density in the left upper mediastinum. A CT guided fine need aspiration (FNA) of the superior mediastinal mass was performed on November 24, 1994 and cytological smears revealed clusters of cells having moderate pleomorphism, individual cells with homogeneous nuclei chromatin, inconspicuous nucleolus and cytoplasm with 


\section{Before treatment}

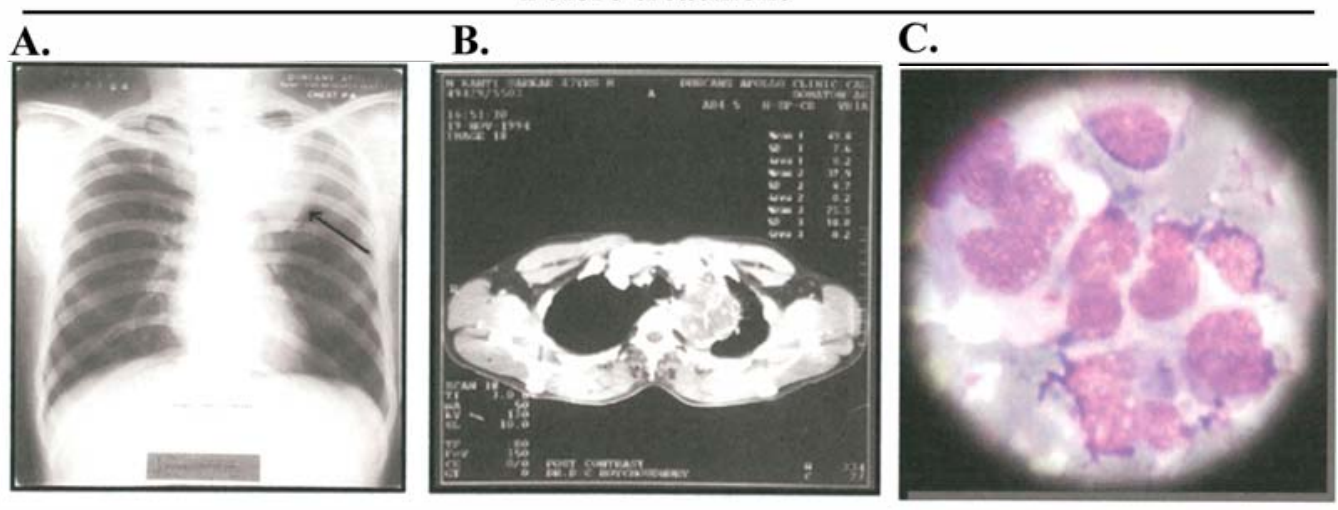

D.

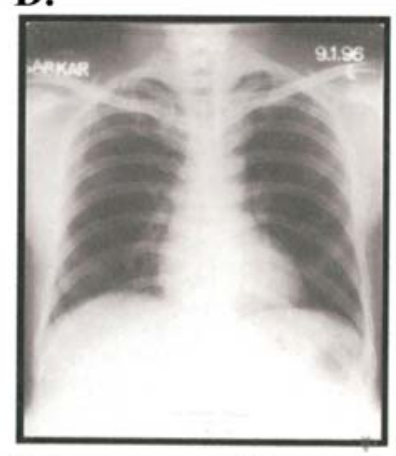

Follow up

E.

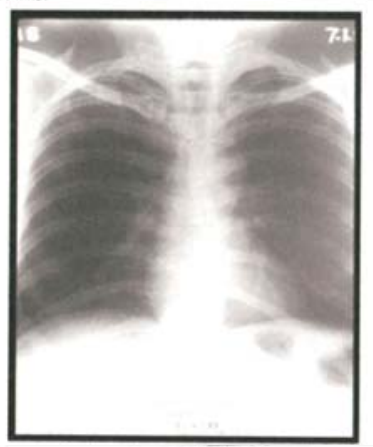

Figure 1. Effect of homoeopathic remedies on lung cancer regression. (A) Chest X-ray exhibits a growth of malignant non-small cell lung carcinoma in the left lung of a 47-year-old male patient. (B) A CT-scan of the chest of the same patient. (C) Histopathology slide of malignant non-small cell lung carcinoma samples collected by FNA from the same patient. (D and E) Chest X-rays show complete regression of tumor growth. Arrow indicates the tumor.

vacuolations supporting the diagnosis of a malignant tumor (Fig. 1C). The NCI Laboratory of Pathology review confirmed the malignancy and categorized it as an adenocarcinoma. According to the American Joint Committee on Cancer Staging (2002), the TNM classification was $\mathrm{T}_{2} \mathrm{~N}_{\mathrm{X}} \mathrm{M}_{0}$ or Stage III cancer.

Treatment. The patient received Kali Carbonicum 200c, a dose of 2 drops, 3 times a week and Ferrum Phosphoricum 3x, 2 tablets twice daily beginning on November 30, 1994 and the treatment continued up to July, 1996. No adverse effects of the therapy were noted. He did not receive conventional cancer therapy.

Follow-up. After 2 months of treatment, the patient gradually became asymptomatic. On January 31, 1995 a chest X-ray revealed considerable shrinkage of the mediastinal mass compared with the initial study. Progressive shrinkage was demonstrated on additional X-rays performed on July 5, 1995 and January 9, 1996 (Fig. 1D). On January 7, 1999, a chest $\mathrm{X}$-ray documented a complete resolution of the mediastinal tumor (Fig. 2E). The patient returned to the clinic every 6 months for follow-up and no reoccurrence has been noted. The date of the last follow-up was September 11, 2006.

Case No. 2. A 77-year-old woman presented to the clinic on September 12, 1994. A chest X-ray performed on December 27, 1994 revealed a wedge shape right upper lobar infiltrate with sharp linear margins. Interstitial markings were prominent in the right lung field. Results of a follow-up chest X-ray on January 9, 1995 demonstrated an unchanged peripheral wedge shaped right upper lobe infiltrate that had an inflammatory appearance. Findings on a January 9, 1995 chest CT scan were initially reported as a dense irregular, lobulated mass with shaggy margins in the posterior segment of the right upper lobe and lateral segment of the right middle lobe from the base to the periphery suggestive of bronchogenic carcinoma. The NIH DRD review did not describe a definitive mass, although a peripheral wedge shaped defect with air bronchograms was noted. A CT-guide FNA of this region of the right lung was performed on January 9, 1995 and the initial pathology report described a small cell type carcinoma of the lung. The NCI Laboratory of Pathology review later reclassified this as an adenocarcinoma. According to the American Joint Committee on Cancer Staging (2002), the TNM classification was $\mathrm{T}_{2} \mathrm{~N}_{\mathrm{x}} \mathrm{M}_{0}$ or Stage II cancer.

Treatment. The patient received Kali Carbonicum 200c, a dose of 2 drops, 3 times a week and Ferrum Phosphoricum 3x, 2 tablets twice daily beginning on January 12, 1995 and treatment continued up to September 1995. No adverse effects of the therapy were noted. The patient did not receive conventional cancer therapy.

Follow-up. After treatment, the patient became asymptomatic after two months. A chest X-ray performed on March 24, 1995 revealed a complete resolution of the consolidation in the right mid zone, residual streaky opacity of fibrosis, adjacent 


\section{Before treatment}

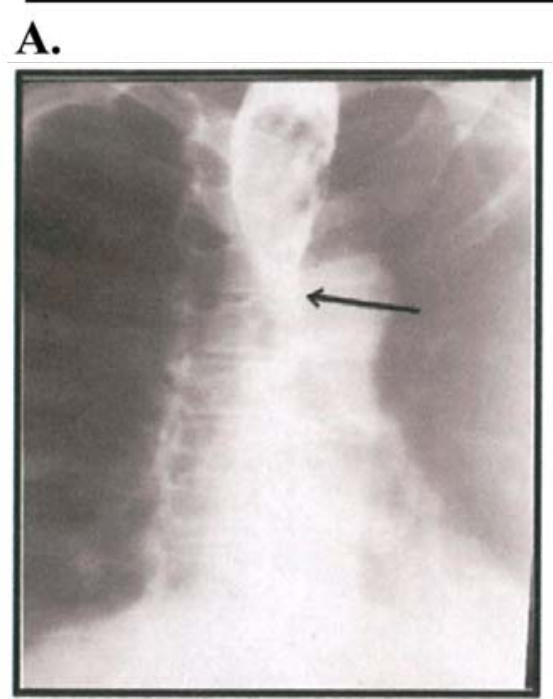

B.

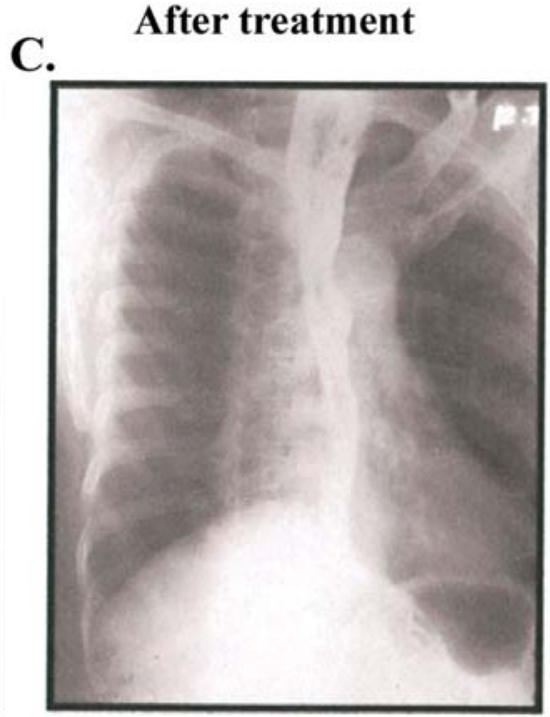

Figure 2. Effect of homoeopathic remedies on oesophageal cancer regression. (A) Barium meal-X-ray exhibits a large proximal filling defect with the dilated portion of the oesophagus of a 75-year-old male patient representing a tumor. (B) A histopathology slide of oesophageal carcinoma samples collected by endoscopy from the same patient. (C) Barium meal-X-ray exhibits a complete regression of the disease. Arrow indicates the tumor.

pleural thickening and prominent bronchovascular markings. An NIH DRD review of this film noted the continued presence of a small wedge shaped lesion consistent with resolving pneumonia. A chest X-ray performed on December 19, 1998 showed residual fibrotic scarring in the right upper lobe though without active disease. The patient returned to the clinic every 6 months for follow-up examinations and thus far no re-occurrence has been seen. The date of the last follow-up was on December 24, 2003.

Case No. 3. A 75-year-old man presented to the clinic with difficulty swallowing, dyspepsia and eructation for the preceding 2 months. On October 17, 1996 an oesophagogram (Fig. 2A) showed a large proximal filling defect within the dilated portion of the oesophagus representing a tumor or another obstruction when reviewed by the NIH/DRD. An endoscopy performed on November 29, 1996, confirmed a tumor with the proximal end $22 \mathrm{~cm}$ into the oesophagus extending to the gastroesophageal junction and causing

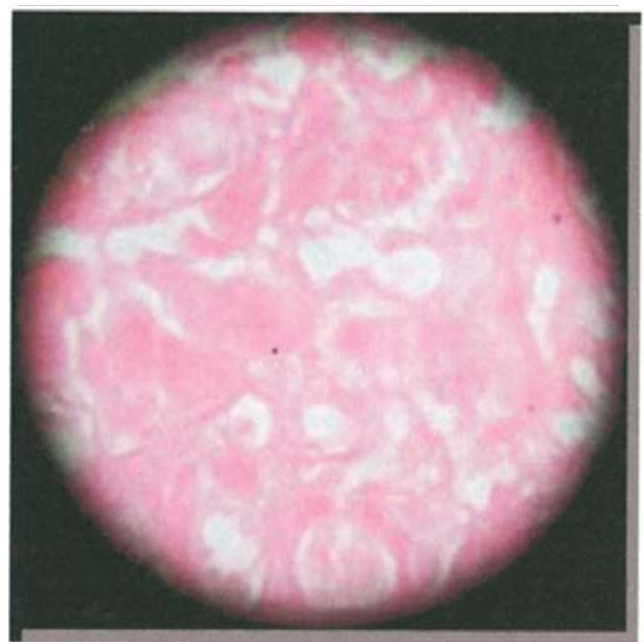

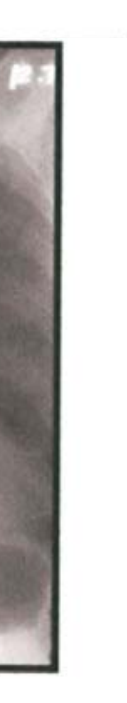


improvement in the patency of the oesophagus compared to the previous oesophagogram dated 17 October, 1996. The NIH DRD review of the films from October 17, 1996 and July 12, 1997 studies noted a persistent though smaller defect in the proximal oesophagus at the site of the previously identified mass with a reduction in the degree of obstruction and return to a normal diameter of the distal oesophagus (Fig. 2C). The patient gradually became asymptomatic and is currently in good health, with no symptoms of dysphagia since July, 1997. The last date of follow-up was in December 1998.

Case No. 4. A 75-year-old woman presented to the clinic with difficulty swallowing and regurgitation of food and fluids while eating for the preceding 3 months. A barium oesophagogram on August 19, 1995 was attempted but not completed due to obstruction. X-ray results showed a gross filling defect at the mid and lower third of the oesophagus with complete obstruction at the lower third of the oesophagus. The NIH DRD review of this film confirmed the presence of a high grade obstruction in the upper oesophagus with an abrupt cut off of the barium column. To permit enteral feedings, the patient underwent a jejunostomy on August 21, 1995. An endoscopic oesophageal biopsy was performed on August 28, 1995. The original pathological report described features suggestive of squamous cell carcinoma. The NCI Laboratory of Pathology review of the specimen revealed adenocarcinoma. Due to the patient's poor general condition, no attempt was made at oesophageal dilatation. According to the American Joint Committee on Cancer Staging (2002), the TNM classification was $\mathrm{T}_{2} \mathrm{~N}_{\mathrm{x}} \mathrm{M}_{1}$ or Stage IIIb possibly Stage IV cancer.

Treatment. The patient received Condurango 30c, 2 drops, twice daily beginning on September 7, 1995 and the treatment continued up to May, 1996. No adverse effects of the therapy were noted. She did not receive conventional cancer therapy.

Follow-up. A barium oesophagogram X-ray was performed on October 12, 1995. The initial report indicated that the contrast media passed freely through the oesophagus with a narrowing at the cardiac end. A curling oesophagus with a diverticula formation was also noted. The NIH DRD review of the film revealed a patent oesophagus with secondary and tertiary contractions suggesting dysmotility though without evidence of a mass or mucosa irregularity. However, the NIH radiologist recommended that an annular oesophageal cancer could be present but not visible given the limited nature of the study ( 2 views only). The patient gradually became asymptomatic since November 1995 with improvement in the dysphagia. The jejunostomy tube was removed on October 12, 1995. The patient returned to the clinic every 6 months for follow-up examinations and no reoccurrence has been seen. The last date of follow-up was January 1999.

\section{Discussion}

Classical homoeopathy uses an extensive patient interview to elicit symptoms and personal characteristics to determine the most appropriate single homoeopathic preparation for that patient (3). The use of a specific homoeopathic medicine for the treatment of a single allopathic diagnosis has been studied in several clinical trials aimed at assessing the validity of these approaches in clinical practice (1,8-11). However, the use of homoeopathic regimens in the treatment of cancer is less well studied (12). Well-designed prospective, randomized, controlled, clinical trials critically evaluating the efficacy of homeopathic therapy for the treatment of cancer have not been performed. Published results from two, well-controlled trials provide the rationale for further investigation of certain homoeopathic preparations in combination with standard therapy for the management of some side effects of conventional cancer therapy $(13,14)$.

Another randomized double-blind placebo-controlled trial performed in Italy included 66 patients with breast cancer who were undergoing chest wall radiotherapy (15). Patients received homoeopathically-prepared belladonna or placebo to determine the effects of therapy on radio-dermatitis. There was a non-significant trend in favour of the belladonna preparation during radiotherapy as well as a significant benefit during the post-radiotherapy recovery period. However the use of a small number of patients as well as a non-validated scale to measure radio-dermatitis weakened the findings of this study.

Several case reports of homoeopathic treatments of cancer have also been published (12). Other homoeopathic physicians have published cases describing their experiences in the treatment of cancer patients $(6,7,16)$. Due to the lack of prospective research or rigorously documented and reported retrospective case studies of the use of so-called 'alternative therapies' for cancer treatment, the NCI developed a program to systematically evaluate retrospective case report data and identify interventions for which there is sufficient evidence to justify NCI-initiated prospective research. The four major criteria for an optimal case submission to this program are: a) definitive diagnosis of cancer at the time the patient received the therapy in question; b) documentation of disease response; c) absence of confounders such as concurrent or recent conventional anticancer therapy; and d) documented treatment history.

This report represents the first detailed description of the results of a review of cases by the NCI. The therapies received by these patients included Kali Carbonicum and Ferrum Phosphoricum for lung cancer and Condurango for oesophageal cancer. Homoeopathic remedies are derived from a variety of substances from plants, minerals and animals. Dilutions of soluble substances are succussed serially to form a mother tincture from which the desired potency is achieved. One part of the mother tincture is mixed with 99 parts of $87 \%$ alcohol and succussed to achieve $1 \mathrm{C}$ potency in the centesimal scale (1:100). Decimal potencies (1 to 10 or $\mathrm{X}$ potencies) are often formed and are weaker than centesimal potencies (17). The homoeopathic preparations used in these cases are not commonly used in classical homoeopathic practice for the treatment of patients with lung cancer. Characteristically, patients in this clinic receive other homoeopathic prescriptions for the management of specific symptoms (e.g. pain, haemoptysis, nausea and vomiting). Unlike other centres in India, the frequency of similar positive outcomes by patients with cancer at this centre in India is clear.

Four case studies out of 12 were initially presented to the NCI BCS Program in 1999 and underwent independent 
confirmation of the diagnosis and radiographic response. The entire case series was also reviewed and commented on by the Cancer Advisory Panel for Complementary and Alternative Medicine. Based on the positive outcomes seen in these case studies, several questions have arisen that have warranted further study. Notably, could the results be replicated and if so what is their frequency? Furthermore, how could a workable protocol be developed to study the use of this approach to homoeopathic management in a prospective clinical trial of a specific type and stage of cancer? In addition, several variables needed to be addressed to ensure validity and reliability of future findings. These areas include providing consistent quality of the pathology and imaging, improvement of patient follow-up, which was inconsistent because of travel issues and incorporating a formal computerized data management system. The greatest barrier to addressing these issues is the significant financial support required due to the extreme poverty in the area where the clinic operates.

In conclusion, this report describes the process and outcome of a selected case series review via the NCI BCS Program. The results of the review were deemed to be sufficient to warrant an NCI-initiated prospective research follow-up in the form of an observational study. A protocol for the conduct of this study has been approved by the NCI Special Studies institutional review board and an application for approval of this project has been submitted to the Indian Council for Medical Research.

\section{Acknowledgements}

We thank Drs Jeffrey D. White, Peter Choyke and Paul Duray, for reviewing the X-rays, CT-scan plates and histopathological slides, respectively, on behalf of the NCI. We would also like to acknowledge the contributions of Dr Satadal Das, in review of the histopathological slides at the PBHRF and Gobinda Chandra Das, for help in preparing the manuscript. We dedicate this study to the memory of Ms. Krishna Banerji.

\section{References}

1. Bark T and Dwyer D: Homeopathy. In: Text Book of Complementary and Alternative Medicine. Yuan CS and Bieber EJ. The Parthenon Publishing Group, Boca Raton, FL, pp65-79, 2003.
2. Carlston M: Homeopathy today. In: Classical Homeopathy. Carlston M and Micozzi MS. Churchill Livingstone, New York, pp33-45, 2003.

3. Gordon D: Homeopathic principles in patient care. In: Classical Homeopathy. Carlston $M$ and Micozzi MS. Churchill Livingstone, New York, pp103-119, 2003.

4. Eisenberg DM, Davis RB, Ettner SL, Appel S, Wilkey S, van Rompay M and Kessler RC: Trends in alternative medicine use in the United States, 1990-1997: results of a follow-up national survey. JAMA 280: 1569-1575, 1998.

5. Hawkins MJ and Friedman MA: National Cancer Institute's evaluation of unconventional cancer treatments. J Natl Cancer Inst 84: 1699-1702, 1992.

6. Lee CO: Homeopathy in cancer care: Part II-Continuing the practice of 'like curing like'. Clin J Oncol Nurs 8: 327-330, 2004.

7. Lee CO: Translational research in cancer complementary and alternative medicine: the National Cancer Institute's Best Case Series Program. Clin J Oncol Nurs 8: 212-214, 2004.

8. Weiner DK and Ernst E: Complementary and alternative approaches to the treatment of persistent musculoskeletal pain. Clin J Pain 20: 244-255, 2004.

9. McCarney RW, Lasserson TJ, Linde K and Brinkhaus B: An overview of two Cochrane systematic reviews of complementary treatments for chronic asthma: acupuncture and homeopathy. Respir Med 98: 687-696, 2004.

10. McCarney RW, Linde K and Lasserson TJ: Homeopathy for chronic asthma. Cochrane Database Syst Rev: CD000353, 2004.

11. Jacobs J, Jonas WB, Jimenez-Perez $M$ and Crothers D: Homeopathy for childhood diarrhea: combined results and metaanalysis from three randomized, controlled clinical trials. Pediatr Infect Dis J 22: 229-234, 2003.

12. Paterson IC: Homeopathy: what is it and is it of value in the care of patients with cancer? Clin Oncol (R Coll Radiol) 14: 250-253, 2002.

13. Oberbaum M, Yaniv I, Ben-Gal Y, Stein J, Ben-Zvi N, Freedman LS and Branski D: A randomized, controlled clinical trial of the homeopathic medication TRAUMEEL $\mathrm{S}$ in the treatment of chemotherapy-induced stomatitis in children undergoing stem cell transplantation. Cancer 92: 684-690, 2001.

14. Yakir M, Kreitler S, Brzezinski A, Vithoulkas G, Oberbaum M and Bentwich Z: Effects of homeopathic treatment in women with premenstrual syndrome: a pilot study. Br Homeopath J 90: 148-153, 2001.

15. Balzarini A, Felisi E, Martini A and De CF: Efficacy of homeopathic treatment of skin reactions during radiotherapy for breast cancer: a randomised, double-blind clinical trial. $\mathrm{Br}$ Homeopath J 89: 8-12, 2000.

16. Ramakrishnan AU and Coulter C: A Homeopathic approach to cancer, St. Louis, MO, Quality Medical Publishing Inc., 2001.

17. Yasgur J: Homeopathy Dictionary, Greenville, PA, Van Hoy Publishers, 1998. 